\title{
A Proposal for the Metrological Characterization of Circular Features with Digital Optical Machines
}

\author{
P. Maresca, E. Gómez, J. Caja, C. Barajas and M. Berzal \\ Technical University of Madrid, Department of Mechanical Industrial Engineering, Ronda de Valencia, \\ 3; 28012 Madrid, Spain.
}

\begin{abstract}
This paper aims to analyze the different adjustment methods commonly used to characterize indirect metrology circular features: least square circle, minimum zone circle, maximum inscribed circle and minimum circumscribed circle. The analysis was performed from images obtained by digital optical machines. The calculation algorithms, self-developed, have been implemented in Matlab $\mathbb{R}$ and take into consideration as study variables: the amplitude of angular sector of the circular feature, its nominal radio and the magnification used by the optical machine. Under different conditions, it was determined the radius and circularity error of different circular standards. The comparison of the results, obtained by the different methods of adjustments used, with certified values for the standards, has allowed us to determine the accuracy of each method and its scope.
\end{abstract}

Keywords: dimensional metrology, optical machines, no contact measurements, circular features, adjustment method.

PACS: 06.20.-f, 06.30.Bp, 06.60.Mr, 89.20.Bb, 89.20.Ff, 89.20.Kk,

\section{INTRODUCTION}

The realization of dimensional measurements in the field of micro and nano manufacturing is usually impracticable by mechanical probing systems, so it is common to use optical devices [1,2]. A special case is constituted by circular features, for example small holes, rounded notches, fillet radii, etc. [3]. Measurement optical instruments allow, from the coordinates of three or more edge pixels, the indirectly determination of the coordinates of the center, the value of the radius and circularity error. The determination of these geometric parameters can be performed through four well-known calculation methods: least square circle (LSC), minimum zone circle (MZC), maximum inscribed circle (MIC) and minimum circumscribed circle (MCC). The literature reviewed shows metrological studies where these methods are applied for the evaluation of circular features starting from points obtained by coordinate measuring machines (CMMs) [4-9]. These studies indicate that the most significant variable in this kind of indirect measurements is the angular separation $(\alpha)$ between the extreme points of scan [10]. So that, by increasing $\alpha$ the calculated diameter $\left(d_{\mathrm{c}}\right)$ converges to the nominal value of the measurand $\left(d_{0}\right)$. In many cases, the physical characteristics of the measurands force to take measurements from small angle values, thereby large deviations of the results and a huge variability are produced. This situation is very similar to what occurs with digital optical machines. From an 
analytical point of view, optical equipment can be considered a three-coordinate measuring which has been stripped of the $Z$ axis. In principle, this circumstance might seem a simplification to the problem; however, despite measuring in only twodimensional, digital optical equipments incorporate additional sources of uncertainty that require a specific study. One of these sources, as it has been said, is the angular separation of the extreme points of measurement $(\alpha)$, but also the density of pixels captured along the circular profile, instrument resolution, magnification, optical aberrations, etc.

Research in the field of numerical computation have allowed the development of different algorithms capable of solving the exposed adjustment models (LSC, MZC, $\mathrm{MCC}$ and $\mathrm{MIC}$ ), according to the discrete approximations mathematical approaches in the literature [8-11]. In this article we employ the Simplex method [11] for solving mathematical methods MZC, MIC and MCC, using the limaçon approximation [1, 12] that allows linearizing the constraints due to the optimization problems. All computation algorithms, self-developed, have been implemented in MatLab ${ }^{\circledR}$. For the resolution of the LSC method a linear parameterization model (LLS), developed through an own algorithm, proposed by the National Physical Laboratory (NPL) [13] is employed. All algorithms developed have been particularized for metrological conditions of the digital optical machines, starting from the coordinates $\left(x_{i}, y_{\mathrm{i}}\right)$ of the pixels detected on the outline of the circular profile.

\section{MATHEMATICAL DEFINITION OF THE PROBLEM}

Mathematically the four methods MCC, MIC, MZC, and LSC can be raised as optimization problems. These methods predict the parameters are calculated through a series of iterations, from the values of the pixels coordinates $\left(x_{i}, y_{i}\right)$, supplied by the user and/or machine. In mathematical terms, it tries to solve the following optimization problems.

\section{Problem}

The center of the reference circle is determined as the center of the circle with minimum radius, $r_{M C C}$, such that all measured points are included inside the circle. Mathematically, it can be written like this:

$$
\left\{\begin{array}{l}
\min r_{M C C} \\
\left(x_{0_{M C C}}-x_{i}\right)^{2}+\left(y_{0_{M C C}}-y_{i}\right)^{2} \leq r_{M C C}^{2}, i=1, \ldots n
\end{array}\right.
$$

\section{MIC Problem}

The center of the reference circle is determined as the center of the circle with maximum radius, $r_{M I C}$, such that all points are outside the circle. Mathematically, it can be written like this: 


$$
\left\{\begin{array}{l}
\max r_{M I C} \\
\left(x_{0_{M I C}}-x_{i}\right)^{2}+\left(y_{0_{M I C}}-y_{i}\right)^{2} \geq r_{M I C}^{2}, i=1, \ldots n \\
\left(x_{0_{M I C}}, y_{0_{M I C}}\right) \in \operatorname{Conv}\left\{\left(x_{i}, y_{i}\right), i=1, \ldots n\right\}
\end{array}\right.
$$

where "Conv" denotes convex hull.

\section{MZC Problem}

The use of this calculation method supposes to find two concentric circles of radius

$r_{\max }$ and $r_{\min }$, inside which are placed all measured points, so that the distance between these two circles is minimum. The problem of minimum zone circle can be written like this:

$$
\left\{\begin{array}{l}
\min \left(r_{\max }-r_{\min }\right) \\
r_{\min }^{2} \leq\left(x_{0_{M Z C}}-x_{i}\right)^{2}+\left(y_{0_{M Z C}}-y_{i}\right)^{2} \leq r_{\max }^{2}, i=1, \ldots n
\end{array}\right.
$$

\section{LSC Problem}

By this criterion, a circle of radius $r_{L S C}$, such that the sum of the squares of the distances of the different points to this circle is minimum is determined. Mathematically, it can be written like this:

$$
\begin{aligned}
& \min \sum e_{i}^{2} \\
& \text { donde } e_{i}=\sqrt{\left(x_{0_{L S C}}-x_{i}\right)^{2}+\left(y_{0_{L S C}}-y_{i}\right)^{2}}-r_{L S C}
\end{aligned}
$$

\section{ALGORITHM FOR DETERMINING MZC, MIC, MCC AND LSC}

Problems MIC, MCC and MZC are essentially problems of optimization, because the constraints are nonlinear functions. Therefore, it is first necessary to convert these restrictions in linear functions. For this it is used a particular geometry called limaçon $[1,12]$. This approach is valid in the presence of small eccentricities, a situation quite common in the area of current study. Using the limaçon approximation the equation of the circle can be linearized, making the calculation algorithm in a trivial process. The distance $\left(r_{i}\right)$ of any point on the circular profile about the origin O (Figure 1) can be calculated as:

$$
r_{i}=x_{0} \cos \theta_{i}+y_{0} \sin \theta_{i}+\sqrt{r_{0}^{2}-\left(x_{0}^{2}+y_{0}^{2}\right) \sin ^{2}\left(\theta_{i}-\phi_{i}\right)}
$$


where $\phi_{i}=\tan ^{-1}\left(y_{0} / x_{0}\right)$.

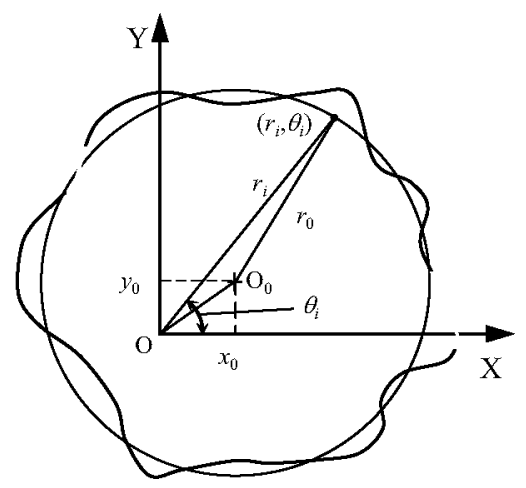

FIGURE 1. Geometric definition of the limaçon approximation.

Applying the limaçon approximation, the equation (5) is approximated as:

$$
r_{i}=r_{0}+x_{0} \cos \theta_{i}+y_{0} \sin \theta_{i}
$$

To demonstrate the limaçon approximation, we refer to paragraph 3.2 of the paper "Maresca et al., Use of coordinate measuring machines and digital optical machines for the geometric characterization of circumference arcs using the minimum zone method, Measurement, 43 (2010), 822-836" [1].

\section{Algorithm for Determining the MZC}

From the limaçon approximation, the algorithm, developed to determine the MZC, can be summarized as following:

$\underline{\text { Step 0: }}$ the iteration counter $j=1$ is initialized.

Step 1: From the coordinates $\left(x_{\mathrm{i}}, y_{\mathrm{i}}\right)$ of $n$ points taken on the circular profile, the least squares circle that fits them is calculated, resulting its center $\left(x_{0 L S C}, y_{0 L S C}\right)$ and radius $r_{L S C}$.

Step 2: The origin of the coordinate system is moved to the obtained center $\left(x_{0 L S C}\right.$, $y_{0 L S C}$ ), obtaining a new reference system $\mathrm{X}_{L S C}, \mathrm{Y}_{L S C}$. Thus, $n$ new pairs of coordinates $\left(x_{i}^{\prime}, y_{i}^{\prime}\right)$ are obtained such that: $x_{i}^{\prime}=x_{i}-x_{0 L S C}$ and $y_{i}^{\prime}=y_{i}-y_{0 L S C}$.

Step 3: The previous Cartesian coordinates are converted to polar coordinates, so that: $r_{i}=\sqrt{x_{i}^{\prime 2}+y_{i}^{\prime 2}}$ and $\theta_{i}=\tan ^{-1} \frac{y_{i}^{\prime}}{x_{i}^{\prime}}$.

Step 4: The optimization problem of minimum zone defined in paragraph 2.3 is solved, applying the limaçon approximation (6) with respect to the new reference system $\mathrm{X}_{L S C}, \mathrm{Y}_{L S C}$ : 


$$
\begin{cases}\min \left(r_{\max _{j}}-r_{\min _{j}}\right) & \\ r_{\max _{j}}+x_{0 M Z C_{j}}^{\prime} \cos \theta_{i}+y_{0 M Z C_{j}}^{\prime} \sin \theta_{i} \geq r_{i}, & \text { para } i \in[1, n] \\ r_{\min _{j}}+x_{0 M Z C_{j}}^{\prime} \cos \theta_{i}+y_{0 M Z C_{j}}^{\prime} \sin \theta_{i} \leq r_{i}, & \text { para } i \in[1, n] \\ r_{\max _{j}}, r_{\min _{j}}>0 & \end{cases}
$$

Step 5: The radius of the minimum zone circle $\left(r_{M Z C j}\right)$ from the values $r_{\max _{j}}$ and $r_{\min _{j}}$ obtained in (7), is determined as:

$$
r_{M Z C_{j}}=\frac{r_{\max _{j}}+r_{\min _{j}}}{2}
$$

Regarding the initial reference system (X, Y) and according to the iteration in question, the coordinates of the center of the minimum zone circle $\left(x_{0 M Z C_{j}}, y_{0 M Z C_{j}}\right)$ are determined as:

If $j=1$

$$
\begin{aligned}
& x_{0 M Z C_{j}}=x_{0 M Z C_{j}}^{\prime}+x_{0 L S C} \\
& y_{0 M Z C_{j}}=y_{0 M Z C_{j}}^{\prime}+y_{0 L S C}
\end{aligned}
$$

If $j \neq 1$

$$
\begin{aligned}
& x_{0 M Z C_{j}}=x_{0 M Z C_{j}}^{\prime}+x_{0 M Z C_{j-1}} \\
& y_{0 M Z C_{j}}=y_{0 M Z C_{j}}^{\prime}+y_{0 M Z C_{j-1}}
\end{aligned}
$$

Step 6: To ensure that the limaçon approximation is valid, we evaluate the distance between the origin of the moved coordinate system used in the $j$-iteration and the center of the minimum zone circle calculated in the same iteration. If this value is zero, the algorithm is terminated, otherwise a new coordinate system with center $\left(x_{0 M Z C j}, y_{0 M Z C_{j}}\right)$ is considered.

So the new coordinates of the points will be $x_{i}^{\prime}=x_{i}-x_{0 M Z C_{j}}$ and $y_{i}^{\prime}=y_{i}-y_{0 M Z C_{j}}$, starting a new iteration $j=j+1$ from step 3 .

\section{Algorithm for Determining the MIC}

For determining the maximum inscribed circle (MIC) and minimum circumscribed circle (MCC) we use the algorithm, performed to determine the $\mathrm{MZC}$, from 0 to 3 steps, having to change the optimization problem from step 4.

Step 4-a: The MIC optimization problem, defined in the paragraph 2.2, is resolved applying the limaçon approximation (eq. 6) with respect to the new reference system $\mathrm{X}_{L S C}, \mathrm{Y}_{L S C}$ : 


$$
\left\{\begin{array}{l}
\min \left(-r_{M I C_{j}}\right) \\
r_{M C_{j}}+x_{0 M C_{j}}^{\prime} \cos \theta_{i}+y_{0 M C_{j}}^{\prime} \sin \theta_{i} \geq r_{i}, \quad \text { para } i \in[1, n]
\end{array}\right.
$$

Step 5-a: Solved the problem of linear programming in the step 4-a, from which we obtain the radii of the MIC, $r_{M I C_{j}}$, the coordinates of the center $\left(x_{0 M I C_{j}}, y_{0 M I C_{j}}\right)$ with respect to the initial reference system $(\mathrm{X}, \mathrm{Y})$ and according to the iteration in question are determined as:

$$
\begin{aligned}
& \text { If } j=1 \\
& x_{0 M I C_{j}}=x_{0 M \pi C_{j}}^{\prime}+x_{0 L S C} \\
& y_{0 M I C_{j}}=y_{0 M I C_{j}}^{\prime}+y_{0 L S C} \\
& x_{0 M C_{j}}=x_{0 M I C_{j}}^{\prime}+x_{0 M I C_{j-1}} \\
& y_{0 M I C_{j}}=y_{0 M C_{j}}^{\prime}+y_{0 M I C_{j-1}}
\end{aligned}
$$

Step 6-a: It follows as in the step 6 of the subparagraph 3.1.

\section{Algorithm for Determining the MCC}

Step 4-b: The MCC optimization problem, defined in the paragraph 2.1, is resolved applying the limaçon approximation (6) with respect to the reference system $\mathrm{X}_{L S C}$, $\mathrm{Y}_{L S C}$ :

$$
\left\{\begin{array}{l}
\min \left(r_{M C C_{j}}\right) \\
r_{M C C_{j}}+x_{0 M C C_{j}}^{\prime} \cos \theta_{i}+y_{0 M C C_{j}}^{\prime} \sin \theta_{i} \leq r_{i}, \quad \text { para } i \in[1, n]
\end{array}\right.
$$

Step 5-b: Solved the problem of linear programming in the step 4-b, from which we obtain the radii of the $\mathrm{MCC}, r_{M C C_{j}}$, the coordinates of the center $\left(x_{0 M C C_{j}}, y_{0 M C C_{j}}\right)$ with respect to the initial reference system $(\mathrm{X}, \mathrm{Y})$ and according to the iteration in question are determined as:

$$
\begin{gathered}
x_{0 M C C_{j}}=x_{0 M C C_{j}}^{\prime}+x_{0 L S C} \\
y_{0 M C C_{j}}=y_{0 M C C_{j}}^{\prime}+y_{0 L S C} \\
\text { If } j \neq 1 \quad x_{0 M C C_{j}}=x_{0 M C C_{j}}^{\prime}+x_{0 M C C_{j-1}} \\
y_{0 M C C_{j}}=y_{0 M C C_{j}}^{\prime}+y_{0 M C C_{j-1}}
\end{gathered}
$$


Step 6-b: It follows as in the step 6 of subparagraph 3.1.

\section{Least Square Method}

For the resolution of this method we use a linear model proposed by the National Physical Laboratory (NPL) [13]. Established the following parameters:

$$
S: \quad x^{2}+y^{2}+a_{1} x+a_{2} y+a_{3}=0
$$

parameters $a_{1}, a_{2}$ and $a_{3}$ are determined by the adjustment method of least squares:

$$
\min _{a} \sum_{i=1}^{n} f_{i}^{2}(a)
$$

We solve the least squares linear problem associated with the function:

$$
f_{i}(a)=x_{i}^{2}+y_{i}^{2}+a_{1} x_{i}+a_{2} y_{i}+a_{3}
$$

expressed in matrix form:

$$
\left(\begin{array}{ccc}
x_{1} & y_{1} & 1 \\
x_{2} & y_{2} & 1 \\
\vdots & \vdots & \vdots \\
x_{n} & y_{n} & 1
\end{array}\right)\left(\begin{array}{l}
a_{1} \\
a_{2} \\
a_{3}
\end{array}\right)=-\left(\begin{array}{c}
x_{1}^{2}+y_{1}^{2} \\
x_{2}^{2}+y_{2}^{2} \\
\vdots \\
x_{n}^{2}+y_{n}^{2}
\end{array}\right)
$$

From $a_{1}, a_{2}$ and $a_{3}$ we obtain the coordinates center $\left(x_{0}, y_{0}\right)$ and radius $\left(r_{0}\right)$ of the circular feature: $x_{0}=-\frac{a_{1}}{2}, y_{0}=-\frac{a_{2}}{2}$ and $r_{0}=\sqrt{\frac{a_{1}^{2}}{4}+\frac{a_{2}^{2}}{4}-a_{3}}$

\section{IMPLEMENTATION OF CALCULATION ALGORITHM}

The algorithm, self-developed, allows (a) obtain the edge of the circular feature from the digital image obtained by the CCD camera, (b) identify the pixels that belong to it and (c) compare the results obtained using the described methods. The algorithm takes as variables: the amplitude of the angular sector of the circular feature, the used amplification, the number of pixels seen on the edge and the nominal radius of the geometry feature. The algorithm has been implemented in MatLab R2007b, along with the efficient library for image analysis, Image Processing Toolbox. Taking into account the work of Midoh et al. [14, 15], we decide to use the edge detection technique based on the Canny filter, which for metrological images obtained in the field of micro and nano manufacturing, is the more effective in terms of probability, certainty and accuracy [16]. Additionally, we have been used the thresholding techniques [17]. Next, the steps of the measurement process are shown and illustrated. 
We have been used as initial image the image of a ring gauge with nominal diameter $d_{\mathrm{n}}=1.497 \mathrm{~mm}$, obtained with a digital optical machine, Tesa Viso 300 .

Step1: The algorithm receives as inputs: (a) the image to be treated (Figure 2), (b) the coordinates of the pixels in the upper left and lower right and (c) the amplitude of the angular sector to be evaluated.

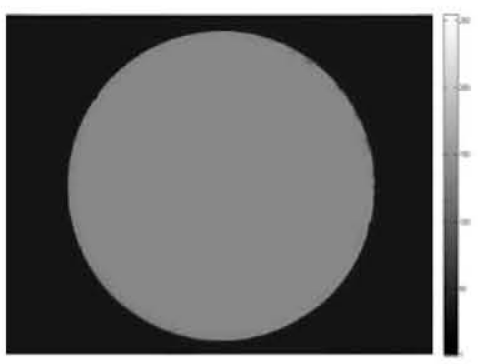

FIGURE 2. Initial image of a ring gauge with nominal diameter $\left(d_{\mathrm{n}}=1.497 \mathrm{~mm}\right)$.

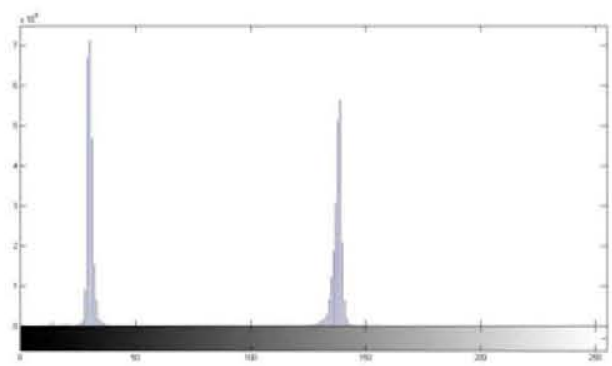

FIGURE 3. Histogram of the initial image.

Step 2: A threshold value using the global threshold method is applied, calculated from the procedure proposed by Gonzalez and Woods [17]. Starting from the histogram of the initial image of the ring gauge (Figure 3 ) we can be determined the threshold coefficient by calculating the mean value $(T)$ between the maximum and minimum intensity values of the image. The image is segmented using the value $T$, so two groups of the pixels are produced; the pixel group, $\mathrm{G}_{1}$, with gray intensity value $\geq T$ and the other one, $\mathrm{G}_{2}$, including pixels with value $<T$. The mean value of gray intensity in each group is calculated, respectively $\mu_{1}$ and $\mu_{2}$ for the groups $\mathrm{G}_{1}$ and $\mathrm{G}_{2}$, and a new threshold value is evaluated as: $T=1 / 2\left(\mu_{1}+\mu_{2}\right)$.

These steps are repeated until the $T$ value in the successive interactions is smaller than a predefined value $T_{0}$, in this case this value is equal to 0.5 .

Step 3: Edge detection is performed from an annulus defined by the user on the image captured by the optical machine (in Figure 4 it is shown in green color). Users also can define the angular sector to be assessed, i.e. the extreme position of the pixels that define the circular shape measured. Then the program generates a rectangular area of evaluation (Figure 4) to which it applies the Canny filter and the threshold value, calculated in the previous step. Then select the edge pixels and determines its coordinates (Figure 5).

Step 4: The least squares circle that best approximates the pixels previously obtained is determined.

Step 5: The coordinates of the edge pixels are converted to polar coordinates with respect to the center of the least squares circle of given in the previous step. 
Step 6: A self-developed filtering process, which allows to eliminate the pixels that have a probability of occurrence less than a certain value defined by the product $\mathrm{k} \sigma$, where $\sigma$ is the sample standard deviation associated with the radius of calculated least square circle and $\mathrm{k}$ is a coverage factor (assuming a normal distribution, the probabilities of occurrence are approximately $65 \%$ for $\mathrm{k}=1$ and $95 \%$ for $\mathrm{k}=2$ ) [1] is applied.

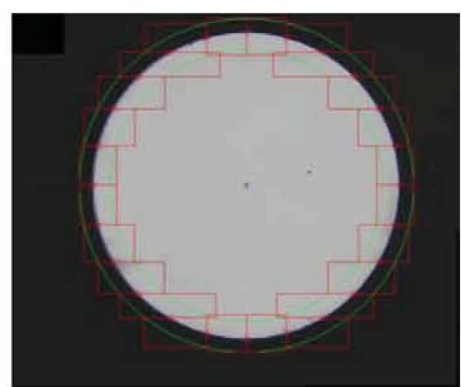

FIGURE 4. Edge assessment zones.

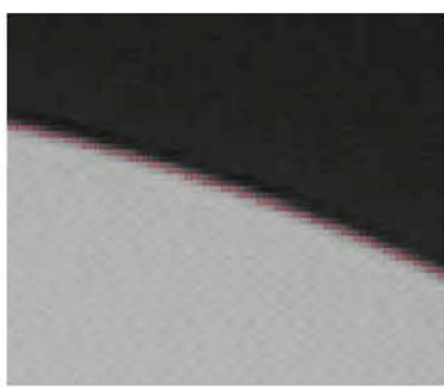

FIGURE 5. Edge pixels detected by Canny.

Step 7: The algorithms for the determination of the LSC, MZC, MCC and MIC ( 3 ) are applied. Images of the circles obtained for the case of complete circumference (Figure 6) and ones of an angular sector of $120^{\circ}$ (Figure 7) are shown.

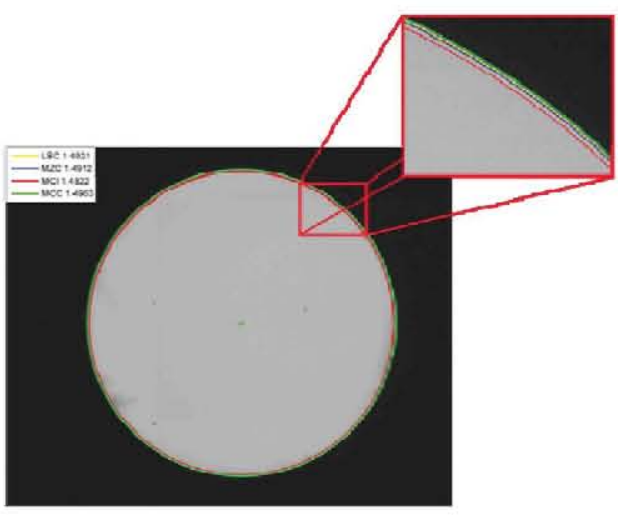

FIGURE 6. Circles calculated for a complete circle.

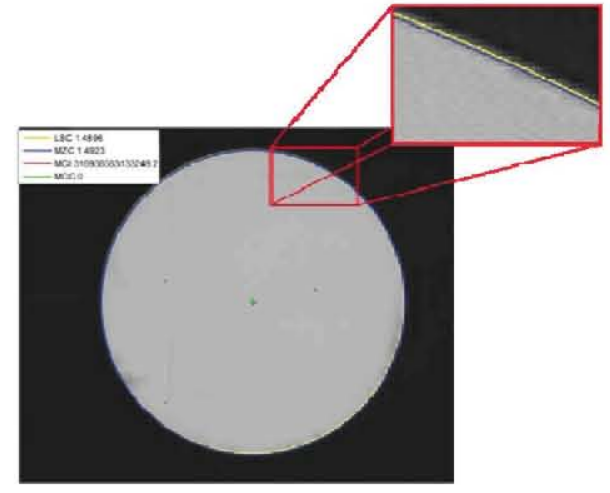

FIGURE 7. Circles calculated for an angular sector of $120^{\circ}$.

\section{RESULTS OF THE COMPARISON BETWEEN THE DIFFERENT METHODS}

In order to demonstrate the applicability and reliability of the proposed calculation algorithm, and compare the results obtained by applying the four adjustment methods (MZC, LSC, MCI, MCC), we consider a ring gauge with nominal radius equal to $0.7485 \mathrm{~mm}$ and a reference disk with nominal radius equal to $1.5 \mathrm{~mm}$, both with certified circularity error equal to $4 \mu \mathrm{m}$. In both cases, the evaluation of the radius was performed varying the amplitude of the angular sector taken on the circular edge. The standards were measured with a digital optical machine TESA VISIO 300, (resolution: 
$1 \mu \mathrm{m}$, magnification: $10 \mathrm{x} \div 130 \mathrm{x}$, vision software: PC-DMIS, $\left.\mathrm{U}_{(\mathrm{k}=2)}=2 \mu \mathrm{m}\right)$ taking into account angular sectors multiples of $30^{\circ}$. In the following figures (Figures 8 and 9) the values of the radius and circularity error of the ring gauge, for different values of the angle $\alpha$ and for the four adjustment method indicated, are shown.

It shows how the radii calculated with the MCC and MIC methods can be evaluated only from $\alpha$ equal to $210^{\circ}$, because for lower values entries received by the algorithm are not sufficient to apply the optimization models described. The values of the radius and circularity error are shown in Figures 10 and 11.

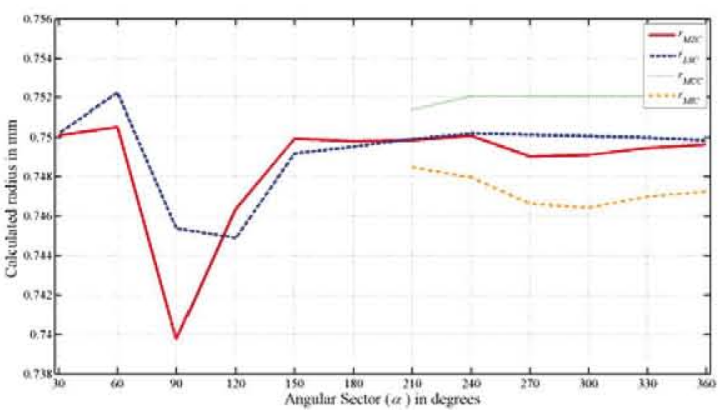

FIGURE 8. Radii calculated of the ring gauge $(\mathrm{r}=0.7485 \mathrm{~mm})$ varying $\alpha$.

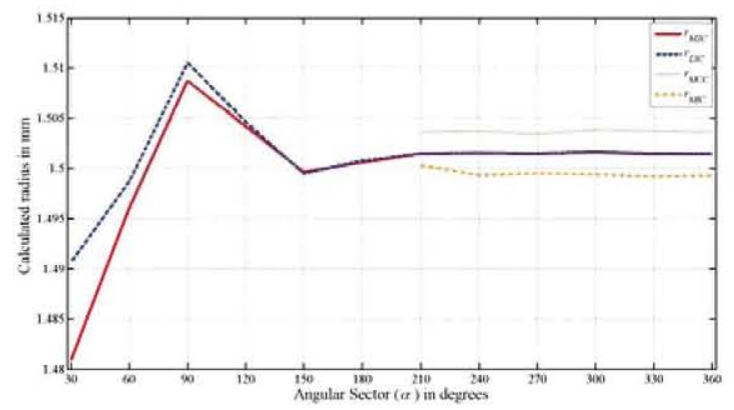

FIGURE 10. Radii calculated of the reference disk $(\mathrm{r}=1.5 \mathrm{~mm})$ varying $\alpha$.

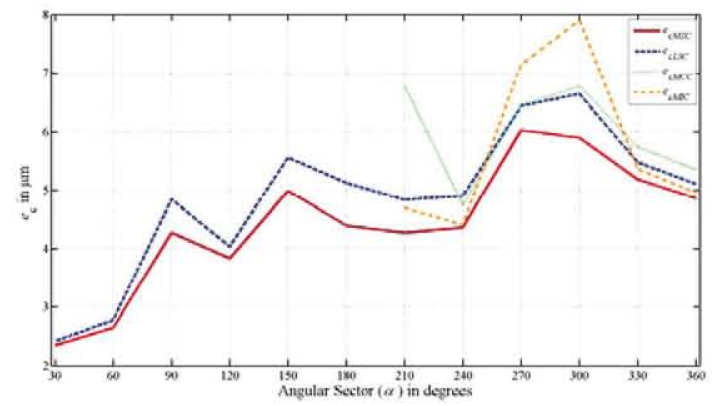

FIGURE 9. Circularity error of ring gauge $(\mathrm{r}=0.7485 \mathrm{~mm})$ varying $\alpha$.

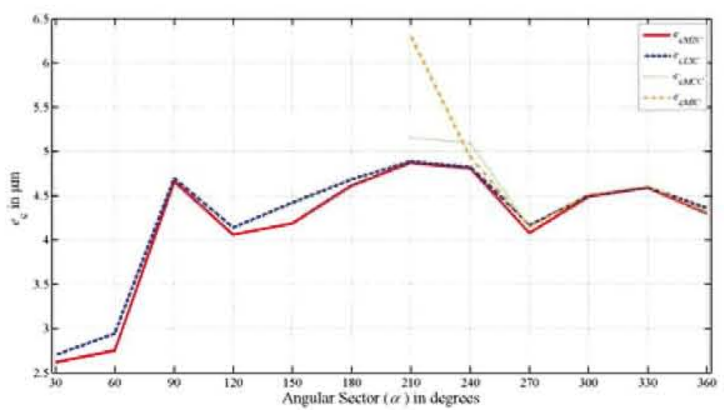

FIGURE 11. Circularity error of the reference disk $(\mathrm{r}=1.5 \mathrm{~mm})$ varying $\alpha$.

\section{CONCLUSIONS}

The algorithm developed in this work allows to automatically evaluate the geometric parameters (coordinates of the center, radius and circularity error) of any circular feature from a digital image. This assessment can be made according to the four most common methods of adjustment: MZC, MIC, MCC and LSC. The first three methods are based on a linear programming model and the limaçon approximation, while for the LSC method we used a linear parameterization model (LLS) proposed by the National Physical Laboratory [13]. In the case of LSC and MZC, the algorithm is robust and consistent to characterize indirectly any circular features, regardless of the amplitude of angular sector considered. In the case of MIC and MCC, as the ASME recommended [18], the algorithm can be used only for evaluation of complete circles. 
An experimental study has been conducted using calibration standards, with satisfactory results. In short, we recommend using proposed algorithm of minimum zone and/or least squares when you want to perform the metrological characterization of arcs in mechanical parts such as: fillet radii, radii of the cutting tools, "U" notches, etc.. Moreover, given satisfactory results by using images obtained through the digital optical machines, the model can be considered of particular interest for the characterization of circular features in environments sub-micro and nano manufacturing, where it is not possible to perform measurements by physical contact.

\section{REFERENCES}

1. P. Maresca, E. Gómez, J. Caja, C. Barajas and M. Berzal, Measurement 43, 822-836 (2010).

2. E. Gómez, J. Caja, P. Maresca, C. Barajas and M. Berzal, Meas. Sci. Technol. 20, 125301 (2009).

3. E. Gomez, J. Caja, C. Barajas, M. Berzal and P. Maresca, "Considerations to the indirect measurement of radius in pieces with profile projector: theoretical study and experimental checking", Proc. MESIC 2007, Madrid (SPAIN), 2007.

4. P. B. Dhanish and J. Matthew, Measurement 39, 522-531 (2006).

5. H. Späth and G. A. Watson, Mathematical Communications 6, 29-38 (2001).

6. M. S. Shunmugam, Int. J. Prod. Res. 24, 413-425 (1986).

7. G. L. Samuel and M. S. Shunmugam, Precis. Eng.-J. Int. Soc. Precis. Eng. Nanotechnol. 24, 251$263(2000)$.

8. P. B. Dhanish, Int. J. Mach. Tools Manuf. 42, 1589-1594 (2002).

9. L. Xiuming and S. Zhaoyao, Precis. Eng.-J. Int. Soc. Precis. Eng. Nanotechnol. 33, 284-290 (2009).

10. S. D. Phillips, B. Borchardt, W. T. Estler and J. Buttress, Precis. Eng.-J. Int. Soc. Precis. Eng. Nanotechnol. 22, 87-97 (1998).

11. T. S. R. Marthy, Precis. Eng. 8, 19-23 (1986).

12. D. G. Chetwynd, Precis. Eng.-J. Int. Soc. Precis. Eng. Nanotechnol. 1, 137-141 (1979).

13. R. M. Barker, M. G. Cox, A. B. Forbes and P. M. Harris, Software Support for Metrology Best Practice Guide No. 4: Discrete Modelling and Experimental Data Analysis, National Physical Laboratory, Teddington, (UK) 2004, Internet Document, URL:

http://resource.npl.co.uk/docs/science_technology/scientific_computing/ssfm/documents/ssfmbpg4. pdf

14. Y. Midoh, K. Nakamae and H. Fujioka, Meas. Sci. Technol. 18, 579 (2007).

15. Y. Midoh, K. Miura, K. Nakamae and H. Fujioka, Meas. Sci. Technol. 16, 477 (2005).

16. G. Iannizzotto and L. Vita, A multiscale turning angle representation of object shapes for image retrieval, Proc. In Visual 99, Amsterdam, (The Netherlands), 1999.

17. R. C. Gonzalez, R. E. Woods and S. L. Eddins, "Digital Image Processing Using MATLAB", Gatesmark Publishing, 2nd Ed., Knoxville (USA), 2009.

18. The American Society of Mechanical Engineers, ASME Y14.5 2009 [Revision of ASME Y14.5M1994 (R2004)] Dimensioning and Tolerancing, The American Society of Mechanical Engineers, New York (USA), 2009. 